
\title{
25 Research Square \\ Validation of Acromegaly Quality of Life Questionnaire (AcroQol) for the Iranian Population
}

\section{Mina Danaei}

social determinants of health research center, institute of futures studies in health, kerman university of medical sciences

\section{Leyla Bahadorizadeh}

antimicrobial resistance research center, institute of immonology and infectious disease, iran university of medical sciences, tehran, iran

\section{Afsaneh Dehnad}

school of health management and informationsciences, iran university of medical sciences, tehran, iran

Shirin Mohamadzadeh

department of english language, alzahra university, tehran, iran

Nahid Hashemi-Madani ( $\sim$ nahid.hashemimadani@gmail.com )

ENDOCRINE RESEARCH CENTER, INSTITUTE OF ENDOCRINOLOGY AND METABOLISM, IRAN

UNIVERSITY OF MEDICAL SCIENCES https://orcid.org/0000-0002-6134-9906

\section{Mohammad E Khamseh}

endocrine research center, institute of endocrinology and metabolism, iran university of medical sciences, tehran, iran

\section{Research}

Keywords: Acromegaly, Quality of life, Disease-specific questionnaire

Posted Date: September 14th, 2021

DOI: https://doi.org/10.21203/rs.3.rs-864640/v1

License: (c) (i) This work is licensed under a Creative Commons Attribution 4.0 International License. Read Full License 


\section{Abstract}

Background: Acromegaly is a chronic disease seriously impacting the patients' quality of life. This study aimed to validate the Acromegaly Quality of Life Questionnaire (AcroQoL) for the Persian-speaking population.

Methods: This cross-sectional study recruited 73 Iranian patients with a confirmed diagnosis of acromegaly. The content validity of the scales was evaluated by an expert panel of eight endocrinologists. Construct validity was assessed by using confirmatory factor analysis. Internal consistency was assessed on the basis of Cronbach's alpha, and a goodness-of-fit (GoF) index was calculated to display whether the model fitted the data.

Results: The content validity index (CVI) and content validity ratio (CVR) yielded values of 0.85 and 0.80 , respectively. The average variance extracted (AVE) from physical and psychological dimensions was 0.520 and 0.462 , respectively, exceeding the minimum criterion of 0.40 . Cronbach's alpha for physical and psychological dimensions equaled 0.868 and 0.866 , respectively, indicating the adequate internal consistency of multiple items for each construct. The subscales' $\mathrm{R}$ square and path coefficient were greater than the recommended threshold (physical dimension: 0.778, psychological dimension: 0.873), demonstrating the suitability of this criterion. Finally, the GoF value of 0.29 indicated the model's moderate fit.

Conclusions: The findings revealed that the Persian version of AcroQoL is of adequate validity and reliability for evaluating the quality of life of Iranian people with acromegaly.

\section{Introduction}

Acromegaly, as a chronic disease, can adversely affect the physical, emotional, and social aspects of patients' life $(1,2)$. Acromegaly which is known to be slow in progression and long in duration, occasionally requires life-long medical treatment. This debilitating disease affects patients' quality of life (QoL) due to the associated conditions, including cardiovascular complications, cerebrovascular events, gonadal dysfunction, impaired glucose tolerance, diabetes, sleep apnea, impaired respiratory function, and colonic neoplasms. It can also cause emotional distress by affecting the patients' physical appearance (3-5). Moreover, if the disease persists, the need for life-long medical therapy or radiotherapy may exacerbate the patients' overall health $(6,7)$.

Several studies have investigated psychological morbidities in patients with acromegaly, indicating that anxiety and insomnia occur in $50 \%$ of these patients (1). General health and appearance are also shown to be particularly and severely affected by acromegaly to scores worse than that for obese patients $(2,8)$. It has also been reported that achieving a good biochemical control of the disease is not necessarily associated with a recovery in QoL $(8,9)$. Thus, QoL should be assessed as an independent outcome in patients with acromegaly, emphasizing the necessity of using an appropriate instrument to accurately assess health-related quality of life (HRQoL) in these patients. 
Disease-specific questionnaires specifically designed for a particular condition are more likely to assess patients' self-perceived status and screen patients requiring further evaluation. The Acromegaly Quality of Life Questionnaire (AcroQoL) is the first acromegaly-specific HRQoL measure originally developed by X. Badia et al. in 2001 (10). It consists of 22 items spread across two dimensions: physical (eight items) and psychological (14 items). The psychological domain is further divided into two sub-dimensions evaluating appearance and the diseases' impact on the patients' personal relationship (10). The AcroQoL was originally developed and validated for the Spanish-speaking population. Since then, it has been translated into some other languages (11). AcroQoL showed a Cronbach alpha of 0.92 in different dimensions, a value that is greater than the minimum required for psychometric standards (0.70) (11). Moreover, it indicated an intra-class correlation (ICC) coefficient ranging from 0.74 to 0.88 in different dimensions, higher than the minimum required (0.70) in all the dimensions (11). This questionnaire has been applied in routine clinics for monitoring patients. AcroQo also has the potential to be used for longitudinal assessment in evaluating the impact of interventions or treatments on the perception of wellbeing in patients with acromegaly.

Additionally, it has been recommended that QoL be annually evaluated as a principal outcome of acromegaly (12). According to the World Health Organization (WHO), QoL should be assessed in the context of the culture and values related to the patients' goals, expectations, standards, and concerns (13). Thus, the instruments applied for HRQoL assessment should be validated for the specific population for whom they are used. Nevertheless, the Persian version of AcroQoL had not been validated for the Iranian suffering from acromegaly. Therefore, we aimed to validate the Persian version of this questionnaire both for use in the clinical practice and for research purposes.

\section{Methods}

This study was a psychometric research conducted on 73 patients with a confirmed diagnosis of acromegaly attending a tertiary pituitary clinic for their routine follow-up from 2019 to 2021. The patients were included in the study if they were Iranian and had adequate literacy to fill out the questionnaire. They were excluded from the study if they were reluctant to participate in the research and could not fill out the questionnaire.

Acromegaly is a rare disease, and determining the sample size by applying the general principles of sampling (i.e., respondent-to-item ratio) yields an inappropriately high number of participants. It is recommended that, for each question in the questionnaire, at least 2-3 participants be considered. Thus, an appropriate psychometric tool should be employed for the studies with a low-to-moderate sample size in order to have a precise analysis. Thus, by using the smart Partial Least Square (PLS) software, and convenience sampling, the questionnaires were distributed among 75 patients with a confirmed diagnosis of acromegaly; eventually, 73 questionnaires were completed by the participants $(14,15)$.

Upon obtaining informed consent, we asked the participants to complete the AcroQoL questionnaire. It is a simple questionnaire designed to be self-administered, but for cases where it cannot be self- 
administered, it can be completed through an interview (11). The participants completed the questionnaire twice, with a minimum two-week interval. For some participants who had difficulty reading, the questionnaire was read by a researcher and completed through an interview.

The AcroQoL questionnaire has been designed specifically for the evaluation of HRQoL in patients with acromegaly. It consists of 22 items spread across two dimensions: physical (eight items) and psychosocial (14 items). The questions are scored on a five-point Likert scale. The responses are categorized as "always, most of the time, sometimes, rarely, never" where the item measures the frequency of the occurrence, and as "completely agree, moderately agree, neither agree nor disagree, moderately disagree, completely disagree" where the item measures the patient's degree of agreement (11). Answers are scored from 1 to 5 ; the response "always" or "completely agree" scores 1 , and the response "never" or "completely disagree" scores 5 . The higher score the participant achieves, the lower the expected impact of acromegaly on HRQoL. The score ranges from 8 to 40 for the physical dimension, and from 14 to 70 for the psychological dimension, and the global score ranges from 22 (worst HRQoL) to 110 (best HRQoL). To standardize the score for the simplification of interpretation, the items can be scored from 0 (worst HRQoL) to 100 (best HRQoL) by using the following formula, where $Y$ stands for the recalculated score, and $\mathrm{X}$ represents the sum of all the item responses within the dimension or study score (min. is the minimum possible score in the studied dimension, and max. is the maximum possible score in the studied dimension).

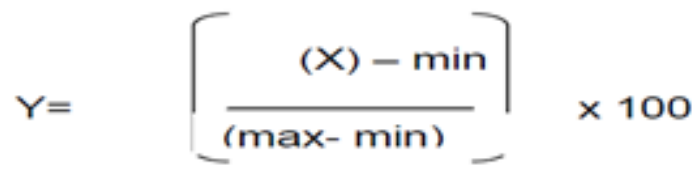

\section{Translation}

We used the Persian version of the AcroQoL questionnaire developed through back-translation by two native-like speakers of the English language, whose first language was Persian. One of the translators translated the original questionnaire into Persian, and the other one back-translated it into English, which was then reviewed by a panel of experts.

\section{Content Validity}

To quantify the content validity of the AcroQoL, eight expert endocrinologists were asked to examine the necessity/precision of each item for the Iranian culture by using a three-point rating scale (essential, useful but not essential, and not essential) and to rate the items of cultural relevancy, clarity, and simplicity. The content validity ratio (CVR) for every item was calculated by using the formula: [Ne- (N/2)] $\div(\mathrm{N} / 2)$ ], where $\mathrm{Ne}$ is the number of panelists choosing "essential" for each particular item, and $\mathrm{N}$ is the total number of panelists. To calculate the content validity index $(\mathrm{CVI})$, the responses were rated from $1=$ not relevant, not simple, and not clear, to $4=$ very relevant, very simple, and very clear. Items with a CVI of $>0.78$ and CVR of $>0.75$ were accepted (16) (17). 


\section{Construct validity}

Construct validity is an important type of validity, showing that the instrument measures what it claims to measure. Using confirmatory factor analysis (CFA), we assessed convergent and divergent validity, representative of the construct validity.

\section{Reliability}

Reliability and internal consistency were evaluated by Cronbach's alpha analysis of the different dimensions of the questionnaire. The time interval for this assessment was at least two weeks. The ICC of $>0.70$ for each domain indicates high reliability and internal consistency (18).

\section{Ethical Considerations}

Having been approved by the Ethics Committee at Iran University of Medical Science (IUMS) (IR.IUMS.REC.1398.526), the study included 73 patients meeting the inclusion criteria. After informing the participants of the purpose of the study and ensuring them about the confidentiality of their information, informed consent was obtained and the questionnaire was completed in two sessions.

\section{Data Analysis}

Descriptive analysis was applied to analyze the patients' characteristics. The AcroQol construct was evaluated via CFA in PLS 3. The outer loadings of the measurement indicators $(>0.70)$ and the average variance extracted (AVE) $(>0.40)$ of the model's constructs were examined to establish convergent validity.

Moreover, the Fornell-Larcker criterion was used to demonstrate divergent validity in the cases when the AVE of a composite construct was higher than the construct's highest squared correlation with any other composite construct (19). Coefficients of $\mathrm{p}$-value and $\mathrm{R}$ square criterion were used to establish the structural model. A greater $\mathrm{R}$ square value for the endogenous structural model indicates a better-fitting model. The weak, medium, and strong fitness of the structural model is determined by $\mathrm{R}$ square standard and path coefficient. As a rough rule of thumb, $R^{2}$ values of $0.75,0.50$, and 0.25 can be described as substantial, moderate, and weak, respectively. The direction and significance of the path coefficient will determine whether the structural model is fit (20). A goodness-of-fit (GoF) index was calculated to display whether the model fits the data (21). SPSS 20 was employed for data analysis, and the results are presented as mean \pm SD (standard deviation) and frequency (percentage).

\section{Results}

This study included 73 patients with acromegaly (response rate: $97.33 \%$ ). The mean $( \pm S D)$ age of the participants was $42.79( \pm 10.53)$ years, and $54.8 \%(n=40)$ of them were women.

\section{Content Validity}


CVI and CVR were applied to assess content validity, which yielded values of 0.85 and 0.80 , respectively, representing good content validity.

\section{Construct Validity}

Convergent validity, tested via CFA, was satisfactory after excluding four questions (questions 8, 16, 17, 18 ) in that all confirmatory factor loadings exceeded 0.5 . The factor loading of all the questions was significant with a range of 0.52 to 0.81 (Fig. 1). In addition, AVE from physical and psychological dimensions was 0.520 and 0.462 , respectively, exceeding the minimum criterion of 0.40 (22). The final instrument included 18 items related to physical $(n=8)$ and psychological $(n=10)$ dimensions. The loading factors for the items on each construct were greater than loadings with all the remaining constructs, and the AVE squared of any construct was greater than its correlation values with other constructs (Fornell and Larcker test), suggesting divergent validity (Tables 1 and 2).

Table 1

Convergent validity and reliability of the Persian version of AcroQoL.

\begin{tabular}{|llll|}
\hline Variables & Cronbach's Alpha & Composite Reliability & Average Variance Extracted (AVE) \\
\hline Physical & 0.868 & 0.896 & 0.520 \\
\hline Psychological & 0.866 & 0.894 & 0.462 \\
\hline
\end{tabular}

Table 2

Divergent Validity of the Persian Version of AcroQoL.

\begin{tabular}{|lll|}
\hline Variables & Psychological & Physical \\
\hline Psychological & 0.680 & - \\
\hline Physical & 0.679 & 0.721 \\
\hline
\end{tabular}

\section{Reliability}

Cronbach's alpha for physical and psychological dimensions equaled 0.868 and 0.866 , respectively, indicating the adequate internal consistency of multiple items for each construct (Table 1) (23). The stability of the scale was examined via a two-week interval test. Based on the $95 \%$ confidence interval of the ICC estimate, values $<0.5$, between 0.5 and 0.75 , between 0.75 and 0.9 , and $>0.90$ are indicative of poor, moderate, good, and excellent stability, respectively (24).

\section{The Structural Model of the AcroQoL}

The subscales' $\mathrm{R}$ square and path coefficient were greater than the recommended threshold (physical dimension: 0.778 , psychological dimension: 0.873 ), suggesting the suitability of this criterion. The t-value derived from bootstrapping in Smart-PLS showed the significance of the effects of variables on one 
another. All the variables were significant at the confidence level of $0.1 \%$. Table 3 presents a summary of the results of testing the structural model of the AcroQoL. Finally, a GoF value of 0.29 indicates the model's moderate fit.

Table 3

Brief results of the AcroQoL (Persian version) structural model.

\begin{tabular}{|lllll|}
\hline Variables & R square & Path coefficient & T value & Significance level \\
\hline Physical & 0.778 & 0.866 & 34.79 & $<0.001$ \\
\hline Psychological & 0.873 & 0.875 & 70.82 & $<0.001$ \\
\hline
\end{tabular}

\section{Descriptive results}

The results of descriptive analyses are demonstrated in Table 4. The mean (SD) of physical and psychosocial dimensions was $57.91( \pm 24.64)$ and $65.71( \pm 22.18)$, respectively.

Table 4

Summary of the descriptive results of AcroQoL (Persian version).

\begin{tabular}{|lll|}
\hline Variables & Mean & SD \\
\hline Physical & 57.91 & 24.64 \\
\hline Psychological & 65.71 & 24.13 \\
\hline Total & 62.25 & 22.18 \\
\hline
\end{tabular}

\section{Discussion}

The results revealed that the AcroQoL questionnaire is a valid and reliable instrument for assessing QoL in the Iranian population with acromegaly. The content validity of the Persian version of AcroQoL questionnaire was approved using both qualitative (i.e., inspection of the expert panel members' comments) and quantitative analysis (i.e., a survey of the level of agreement among expert panel members). CVR, indicative of the necessity of the domain's items, and CVI, representative of simplicity, relevancy, and clarity of the scale's items, were at the acceptable level $(16,17)$. After developing the AcroQoL questionnaire, the developers evaluated its cross-sectional and longitudinal construct validity compared to the other HRQoL instruments and the General State of Health $(25,26)$, although CVI and CVR were not evaluated in those studies.

The results of the CFA were promising. AVE from all the constructs exceeded the minimum criterion of 0.40 , suggesting that the indicators are more strongly related to their specific construct than to other constructs. Therefore, the divergent validity was verified. However, this was the first study to apply the PLS approach for validation of such a disease-specific questionnaire in patients with acromegaly; thus, 
comparison with the results of other studies is difficult. The translated AcroQol questionnaire demonstrated good internal consistency; the values of Cronbach's alpha for the total score and each subscale were all above the recommended threshold of 0.70 , showing high reliability and internal consistency. These results are in line with the findings of the first study reporting the development of the questionnaire. Both the total questionnaire and the two dimensions had a Cronbach's alpha of $>0.80$ (10). Moreover, previous studies demonstrated significant correlations between the entire questionnaire and each dimension of AcroQoL and the generic questionnaires (2). In addition, test-retest reliability, during a minimum two-week interval, demonstrated good stability, similar to the original version of the questionnaire (11).

The GoF indicators used for establishing the structural model demonstrated values of $>0.75$, demonstrating the substantial fit of the model. Finally, the QoL in this population of patients with acromegaly was in line with the results of previous studies, showing that psychological status has a greater impact on QoL in this population (27).

Applying disease-specific instruments to evaluate the QoL in a specific patient population is of significant advantage as these instruments measure aspects specific to the population in question. There are isolated reports of the effect of this chronic disease on Iranian patients' QoL (28); nevertheless, these studies applied general health-related questionnaires. Thus, the validation of the AcroQoL questionnaire is essential for both the annual assessment of the patients in the clinical practice, and for research purposes.

\section{Strengths And Limitations}

This was the first study validating the Persian version of the AcroQoL questionnaire in the Iranian population with acromegaly. The AcroQoL is a comprehensive questionnaire assessing a variety of physical and psychosocial aspects that may affect QoL in these patients. In addition to the consistent data collection method applied in this study, the participants were recruited from a tertiary center presenting socio-cultural variety, thereby making the results more generalizable to different subgroups of the population. However, this study was limited by the small number of patients and the use of convenience sampling.

\section{Conclusion}

The AcroQol questionnaire, showing to possess appropriate reliability and validity, can be completed in both self-administered and interview-based manners, and is simple to score and interpret. Therefore, it seems to be a suitable instrument for predicting the QoL in the Persian-speaking population with acromegaly.

\section{Abbreviations}


AcroQoL: Acromegaly Quality of Life Questionnaire

AVE: average variance extracted

CFA: confirmatory factor analysis

CVI: content validity index

CVR: content validity ratio

GoF: goodness-of-fit

HRQoL: health-related quality of life

ICC: intra-class correlation

QoL: quality of life

\section{Declarations}

\section{Ethics approval and consent to participate}

The study was approved by the ethics committees at Iran University of Medical Sciences (IUMS) (IR.IUMS.REC.1398.526). Informed consent was obtained from all participants.

\section{Availability of data and materials}

The datasets generated and/or analyzed during the current study are not publicly available due [REASON WHY DATA ARE NOT PUBLIC] but are available from the corresponding author on reasonable request.

\section{Conflict of interest}

All authors declare that they have no conflict of interest.

\section{Funding}

No funding was received.

\section{Author's contribution}

MD cleaned and analyzed the data and interpreted results, LB collected data, AD and SHM contributed in the study design and revised the manuscript, NHM contributed in the study design and wrote the manuscript, MEK critically revised the manuscript.

\section{Acknowledgement}

We really appreciate the kind support of Xavier Badía and Susan Webb. 


\section{References}

1. Szcześniak DM, Jawiarczyk-Przybyłowska A, Matusiak Ł, Bolanowska A, Maciaszek J, Siemińska M, et al. Is there any difference in acromegaly and other chronic disease in quality of life and psychiatric morbidity? 2017;68(5):524-32.

2. Webb SM, Badia XJEjoe. Validity and clinical applicability of the acromegaly quality of life questionnaire, AcroQoL: a 6-month prospective study. 2006;155(2):269-77.

3. Giustina A, Chanson P, Bronstein M, Klibanski A, Lamberts S, Casanueva F, et al. A consensus on criteria for cure of acromegaly. 2010;95(7):3141-8.

4. Davi MV, Dalle Carbonare L, Giustina A, Ferrari M, Frigo A, Cascio VL, et al. Sleep apnoea syndrome is highly prevalent in acromegaly and only partially reversible after biochemical control of the disease. 2008;159(5):533-40.

5. Rokkas T, Pistiolas D, Sechopoulos P, Margantinis G, Koukoulis GJWjogW. Risk of colorectal neoplasm in patients with acromegaly: a meta-analysis. 2008;14(22):3484.

6. Kauppinen-Mäkelin R, Sane T, Sintonen H, Markkanen H, Välimäki MJ, Löyttyniemi E, et al. Quality of life in treated patients with acromegaly. 2006;91(10):3891-6.

7. Jagannathan J, Sheehan JP, Pouratian N, Laws Jr ER, Steiner L, Vance MLJN. Gamma knife radiosurgery for acromegaly: outcomes after failed transsphenoidal surgery. 2008;62(6):1262-70.

8. Rowles SV, Prieto L, Badia X, Shalet SM, Webb SM, Trainer PJJTJoCE, et al. Quality of life (QOL) in patients with acromegaly is severely impaired: use of a novel measure of QOL: acromegaly quality of life questionnaire. 2005;90(6):3337-41.

9. Ben-Shlomo A, Sheppard M, Stephens J, Pulgar S, Melmed SJP. Clinical, quality of life, and economic value of acromegaly disease control. 2011;14(3):284-94.

10. Webb S, Prieto L, Badia X, Albareda M, Catala M, Gaztambide S, et al. Acromegaly Quality of Life Questionnaire (ACROQOL) a new health-related quality of life questionnaire for patients with acromegaly: development and psychometric properties. 2002;57(2):251-8.

11. Badia X, Webb SM, Prieto L, Lara NJH, outcomes qol. Acromegaly quality of life questionnaire (AcroQoL). 2004;2(1):1-6.

12. Melmed S, Casanueva F, Klibanski A, Bronstein M, Chanson P, Lamberts $S$, et al. A consensus on the diagnosis and treatment of acromegaly complications. 2013;16(3):294-302.

13. Skevington SMJJopr. Measuring quality of life in Britain: introducing the WHOQOL-100. 1999;47(5):449-59.

14. Alavi MJIJoME. Structural equation modeling (SEM) in health sciences education researches: an overview of the method and its application. 2013;13(6):519-30.

15. Davari A, Rezazadeh AJTJU. Structural equation modeling with PLS. 2013;215(2):224.

16. Polit DF, Beck CT, Owen SVJRin, health. Is the CVI an acceptable indicator of content validity? Appraisal and recommendations. 2007;30(4):459-67. 
17. Ayre C, Scally AJJM, counseling ei, development. Critical values for Lawshe's content validity ratio: revisiting the original methods of calculation. 2014;47(1):79-86.

18. Bernstein IH. Psychometric theory: Tata McGraw-Hill Education; 2010.

19. Vinzi VE, Chin WW, Henseler J, Wang H. Handbook of partial least squares: Springer; 2010.

20. Henseler J, Ringle CM, Sinkovics RR. The use of partial least squares path modeling in international marketing. New challenges to international marketing: Emerald Group Publishing Limited; 2009.

21. Henseler J, Sarstedt MJCs. Goodness-of-fit indices for partial least squares path modeling. 2013;28(2):565-80.

22. Fornell C, Larcker DFJJomr. Evaluating structural equation models with unobservable variables and measurement error. 1981;18(1):39-50.

23. Henseler J, Ringle CM, Sarstedt MJJotaoms. A new criterion for assessing discriminant validity in variance-based structural equation modeling. 2015;43(1):115-35.

24. Koo TK, Li MYJJocm. A guideline of selecting and reporting intraclass correlation coefficients for reliability research. 2016;15(2):155-63.

25. Paisley AN, Rowles SV, Roberts ME, Webb SM, Badia X, Prieto L, et al. Treatment of acromegaly improves quality of life, measured by AcroQol. 2007;67(3):358-62.

26. Trepp R, Everts R, Stettler C, Fischli S, Allemann S, Webb SM, et al. Assessment of quality of life in patients with uncontrolled vs. controlled acromegaly using the Acromegaly Quality of Life Questionnaire (AcroQoL). 2005;63(1):103-10.

27. Bernabéu I, Fajardo C, Marazuela M, Cordido F, Venegas EM, de Pablos-Velasco P, et al. Effectiveness of lanreotide autogel $120 \mathrm{mg}$ at extended dosing intervals for acromegaly. 2020;70(3):575-83.

28. Asadi-Lari M, Sadeghipour A, Mahouzi L, Solaimani Dodaran M, Fallah A. Assessment of the Demographic Characteristics and the Quality of Life in Patients with Pituitary Adenoma in a Referral Pituitary Center in Tehran in 2011. JRUMS. 2015; 13 (8): 695-704.

\section{Figures}




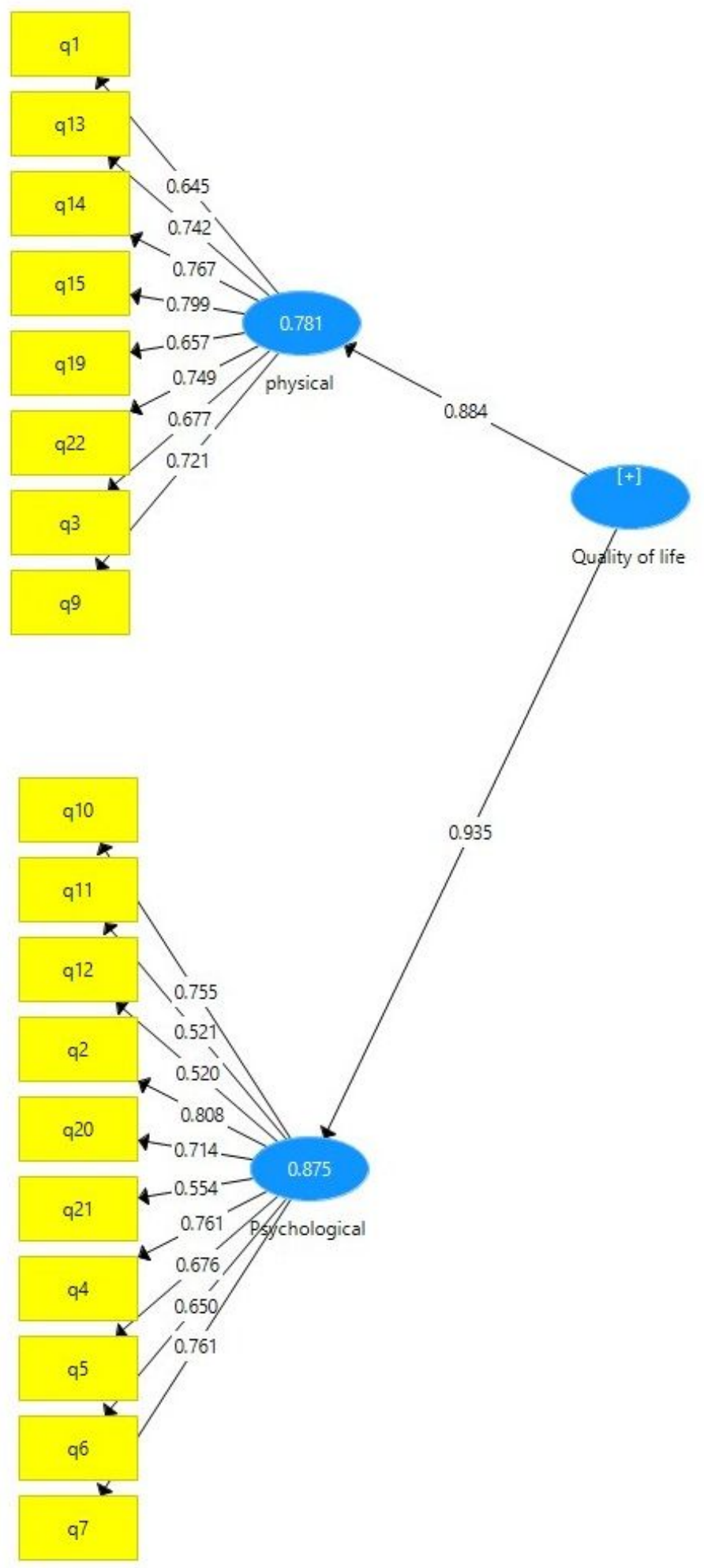

Figure 1

Confirmatory Factor Analysis of the Persian Version of AcroQoL

\section{Supplementary Files}

This is a list of supplementary files associated with this preprint. Click to download. 
- persianversianofthequestionaire.docx 